\title{
UN MODELO PARA LA ESTIMACIÓN DEL ÁREA FOLIAR EN TRES ESPECIES FORESTALES DE FORMA NO DESTRUCTIVA
}

\section{A NON-DESTRUCTIVE MODEL FOR ESTIMATING LEAF AREA INTHREE FOREST SPECIES}

\author{
Marco Cabezas-Gutiérrez \\ Fernando Peña \\ Héctor William Duarte \\ José Fernando Colorado \\ Rodrigo Lora Silva ${ }^{1}$
}

\section{RESUMEN}

Se realizó un estudio de relación entre el área foliar individual y medidas lineares de la hoja de aliso (Alnus acuminata H.B.K) familia Betulaceae, magle (Escallonia pendula Pers.) familia (Escalloniaceae) y roble (Quercus humboldtii Bompland) familia Fagaceae, en condiciones de campo. El objetivo de este estudio fue comparar la eficacia de los modelos lineales, producto de mediciones directas no destructivas del área de la hoja, para luego realizar una validación, a partir de un nuevo conjunto de datos, en condiciones diferentes. El análisis de regresión del área en función del largo de la hoja (Lh), del ancho máximo de la hoja (Ah) y del producto del largo por el ancho (LhxAh) condujo a proponer modelos que pueden ser usados para la estimación apropiada del área foliar individual de la hoja. Para todas las especies, el mejor modelo fue de forma $A F=a+b(L h x A h)$, con coeficientes de determinación superiores a 0,95. En la validación de los modelos, se encontró que la relación entre el área de la hoja media y el área estimada, mediante la respectiva ecuación, fue significante al nivel de $0,1 \%$ y el $r^{2}$ fue de 0,99 en todos los casos. Se concluye, que los modelos lineales encontrados para estimar el área foliar de A. acuminata, E. pendula y $Q$. humboldtii pueden ser usados en estudios biológicos,

${ }^{1}$ Docentes investigadores. Facultad de Ingeniería Agronómica. U.D.C.A. Dirección para correspondencia: Calle 222 No. 55-37, Bogotá D.C. mcabezas@udca.edu.co. agronómicos, ecofisiológicos, ambientales y forestales, con alto grado de precisión y rapidez.

Palabras clave: Área foliar, forestales, regresión lineal, modelos.

\section{SUMMARY}

Relationship studies were carried out between leaf area and linear measurements of leaves of alder (Alnus acuminata H. B. K) (Betulaceae), magle (Escalonia pendula Pers.) (Escalloniacae) and oak (Quercus humboldtii Bompland) (Fagaceae), under open field conditions. The experiment was aimed to compare existing predictive linear leaf area models using non destructive measurements and assessing the accuracy of the optimum model selected from a new data set, under different conditions. The regression analyses of leaf length (Ll), leaf width (Lw) and the product LlxLw revealed several models that could be used to estimate the individual leaf area. For all species the best model was $L a=a+b(L l x L w)$, with the upper determination coefficients being 0.95 . When validating the models it was found that the relationship between the actual leaf area and the predicted one, given by the developed equation was significant at level of $0.1 \%$ with a $\mathrm{r}^{2}$ of 0.99 for all the cases. In conclusion, the linear model, found for the leaf area estimation of $A$. acuminata, E. pendula y Q. humboldtii can be used in biological, agronomical, environmental, ecophysiological and forestry research with high accuracy and rapidity. 
Key words: Leaf area, forestry, linear regression, models.

\section{INTRODUCCIÓN}

Las mediciones del área foliar (AF) son parte fundamental de la investigación en fisiología vegetal, en la agricultura y en la dendrología (Broadhead et al. 2003). El área foliar está asociada con la mayoría de procesos agronómicos, biológicos, ambientales y fisiológicos, que incluyen el análisis de crecimiento, la fotosíntesis, la transpiración, la interceptación de luz, la asignación de biomasa y el balance de energía (Kucharik et al. 1998). Los fisiólogos vegetales, los biólogos y los agrónomos demostraron la importancia del área foliar en la estimación de crecimiento vegetal, en la determinación de etapas fenológicas, en la estimación del potencial de rendimiento biológico y agronómico, en el cálculo del uso eficiente de la radiación solar, como también en el cálculo del uso eficiente del agua y de la nutrición mineral (Sonnentag et al. 2008).

Medir la superficie foliar de un elevado número de hojas puede ser costoso, engorroso y de alto consumo de tiempo. Varios métodos son propuestos para la medición del área de las hojas, pero en ocasiones, ésta se hace difícil, por cuanto los sitios experimentales se encuentran alejados de los centros de investigación y las condiciones de desplazamiento, tiempo, clima y recursos, dificultan el muestreo destructivo, o porque el uso de instrumentación sensible es de alto costo (Ross, 1981; Ajayi, 1990). Esto induce a cometer errores en los cálculos y hace que se desvirtúen los procedimientos.

La determinación del área foliar, se puede realizar por métodos directos e indirectos. Dentro del primer grupo, se destacan las mediciones de hojas a partir de siluetas formadas por los bordes, las cuales, son estampadas en papel y, luego, se miden sus atributos, ya sea por la relación peso:área de figuras circulares o cuadradas, o por fotocopiado sobre papel milimetrado; este método ha sido usado, históricamente, con altos niveles de precisión y con facilidades de realizarlo sin muestreo destructivo (Jonckheere et al. 2004; Ross, 1981). Sin embargo, la mayor desventaja consiste en que la medición es casi imposible de realizar en hojas divididas y con figuras irregulares; tampoco es aplicable para acículas de coníferas, aunque sirve como referencia para probar otras metodologías (Roberts et al. 2005).
Existen métodos estándares, donde las hojas se aproximan a figuras geométricas regulares, cuyas áreas son fáciles de calcular (Kucharik et al. 1998; Levy \& Jarvis, 1999). La alta velocidad para realizar mediciones es la principal ventaja, pero tienen la dificultad de ser muy poco precisos y la subjetividad a la hora de escoger la forma geométrica adecuada en hojas, que, por lo general, son irregulares. Esto conlleva a que las mediciones no representen la realidad del tamaño del área foliar de la planta.

Los planímetros y las cámaras digitales son usados en la actualidad como métodos indirectos de medición del área foliar (Eriksson et al. 2005; Jonckheere et al. 2004). Los primeros presentan ciertas ventajas, como rapidez para obtener datos y alta eficiencia en el procesamiento de muestras, pero no son precisos, debido, principalmente, a las propiedades ópticas de la hoja y la dependencia de fuentes de energía. Cuando los instrumentos son estáticos, se requiere de muestreo destructivo y en algunos casos, cuando son portables, no pueden ser usados con hojas de gran tamaño en la lámina foliar (Beerling $\&$ Fray, 1990). El mayor problema de las imágenes tomadas con cámara digital es el ángulo foliar, el cual, en la mayoría de ocasiones, no es totalmente plano, hecho que produce altos niveles de error procedimental (Craig-Macfarlane et al. 2007).

Los métodos de cálculo consisten en aproximar en alto grado el área de la hoja, mediante regresiones de tipo lineal. También pueden ser relaciones logarítmicas, exponenciales o de otro tipo, donde la medición de un atributo, ya sea el largo de la lámina foliar, el ancho de la hoja o los dos combinados, representen un factor altamente dependiente, que al ser multiplicado por un coeficiente, arroje como resultado el valor muy cercano al real (Peksen, 2007; Cittadini \& Peri 2006; Nautival et al. 1990). Normalmente, se realizan pruebas estadísticas de alta sensibilidad para validar el modelo (Demirsoy et al. 2005). Este método tiene enormes ventajas, puesto que reduce el muestreo destructivo, permite valorar un mayor número de muestras y no depende de equipos ni condiciones, como electricidad y energía de trabajo.

En general, los procedimientos de estimación del área de la hoja involucran medidas de longitud de la lámina foliar, longitud del limbo, ancho de la hoja y la mezcla de estos atributos, mediante la correlación de dichas características con el área de la hoja, producto 
de un análisis de regresión (Gutezeit, 2000; Beerling E Fray, 1990). En la medida en que el método sea cuidadosamente probado y validado, se permite su empleo en diversas situaciones, proporcionando facilidad para la obtención de datos confiables y repetibles en el tiempo.

Existen numerosos trabajos, en donde se demuestra la objetividad y la utilidad del método, especialmente, en cultivos semestrales (Peksen, 2007; De Swart et al. 2004; Bange et al. 2000; Gutezeit, 2000; Panta \& NeSmith, 1994), en cultivos hortícolas (Gutiérrez $E$ Lavin, 2000; Cittadini \& Peri, 2006; Mendoza de Gyves et al. 2007) y en algunas plantas leñosas (Singh, 2007 Broadhead et al. 2003; Peper E McPherson, 2003; Arcila, 1991, pero no en especies forestales tropicales adaptadas a clima frío.

El presente trabajo tuvo como fin determinar un método rápido, fácil y de bajo costo para medir el área foliar de aliso (Alnus acuminata H.B.K) familia Betulaceae, magle (Escallonia pendula Pers) familia Escalloniaceae y roble (Quercus humboldtii Bompland) familia Fagaceae, especies forestales de interés en programas de reforestación, manejo de erosión, captura de carbono y producción de oxígeno en el trópico alto.

\section{MATERIALES Y MÉTODOS}

Entre septiembre y octubre de 2007 y de marzo a abril de 2008, se seleccionaron plantas de $A$. acuminata, E. pendula y $Q$. humboltii de tres años de edad, en las localidades de Tenjo (Cundinamarca) y Firavitoba (Boyacá). En Tenjo, se usó un lote reforestado con estas especies, el cual, está localizado a $2780 \mathrm{msnm}$, con temperatura media del aire de $13^{\circ} \mathrm{C}$, precipitación de $900 \mathrm{~mm}$ anuales y $1100 \mu \mathrm{mol} \cdot \mathrm{m}^{-2} \cdot \mathrm{s}^{-1}$ de fotones como flujo fotónico fotosintético (FFFT). En Firavitoba, se emplearon árboles procedentes de una finca reforestada, ubicada a $2450 \mathrm{msnm}$, con temperatura media del aire de $16^{\circ} \mathrm{C}, 750 \mathrm{~mm}$ de precipitación media anual y $1540 \mu \mathrm{mol} \cdot \mathrm{m}^{-2} \cdot \mathrm{s}^{-1}$ de FFFT. De cada localidad, se tomaron 100 hojas por especie, de tamaño y edad variables, provenientes de 20 árboles, escogidos al azar. Se colocaron en bolsas plásticas y, luego, se pasaron a neveras portátiles y se condujeron al laboratorio de Fitotecnia de la Universidad de Ciencias Aplicadas y Ambientales U.D.C.A, con base en la metodología empleada por Mendoza de Gyves et al. (2007) y De Swart et al. (2004).

A cada hoja, se le determinó el área foliar, mediante un analizador portátil tipo CID 202 Portátil Leaf Área Meter, con $0,1 \mathrm{~mm}^{2}$ de resolución y se midió el largo de la lámina foliar. A las hojas de $A$. acuminata y $Q$. humboldtii se les retiró el pecíolo y, solamente, se tuvo en cuenta la longitud del limbo. En el caso de $E$. pendula, se midió el largo total de la hoja, por su característica semi-sésil. El ancho, se registró en tres puntos de la parte más amplia de la lámina foliar y se promedió.

Con los datos de largo de la lámina foliar (Lh), ancho máximo de la lámina foliar (Ah), el producto del largo por el ancho (LhxAh), se procedió a realizar los respectivos análisis de regresión lineal, para las variables en referencia respecto al área individual de la hoja, mediante el uso del programa EXEL de Microsoft Office 2007® (Samuels E Witmer, 2003). A partir de los resultados encontrados, se escogieron los modelos que presentaron los mayores coeficientes de determinación $\left(R^{2}\right)$ y los menores cuadrados medios del error (CME). Para las tres especies, el producto LhxAh fue el escogido tal y como lo muestra la tabla 1.

Una vez seleccionados los modelos de mayor grado de precisión y de validez biológica, se tomaron 100 hojas de cada especie, con tamaños y edades distintas; en este caso y con el fin de aplicar lo encontrado en condiciones ambientales diferentes, las plantas utilizadas fueron seleccionadas del área de influencia del campus universitario de la U.D.C.A, localizado a $2560 \mathrm{msnm}$, con temperatura media del aire de $14^{\circ} \mathrm{C}$, flujo fotónico fotosintético promedio de $1250 \mu \mathrm{mol}$. $\mathrm{m}^{-2} \cdot \mathrm{s}^{-1}$ y precipitación de $890 \mathrm{~mm}$ anuales. Mediante muestreo destructivo, se midió el área foliar, se tomaron los atributos de largo y ancho de la hoja en cada caso. Se aplicó el modelo $\mathrm{y}=\mathrm{a}+\mathrm{b}($ LhxAh) y mediante la sustitución de valores, se calculó el área foliar estimada, procedimiento empleado en otras especies, por AkramGhaderi E Soltani (2007); Rouphael et al. (2006) y Demirsoy et al. (2005).

Posteriormente, los datos de área foliar estimada y área foliar obtenida por medición directa, se llevaron a un análisis de regresión lineal, para determinar el grado precisión de los modelos. En este caso también, se aplicó el paquete EXCEL 2007, de Microsoft Office®. 
Tabla 1. Coeficientes a y b de los modelos usados para estimar el área foliar de $A$. acuminata $(A)$, E. pendula $(E)$ y $Q$. humboldtii $(Q)$, a partir de la longitud (Lh) y ancho máximo (Ah) de hojas individuales.

\begin{tabular}{|c|c|c|c|c|c|}
\hline \multirow{2}{*}{ MODELO } & FORMA & \multicolumn{3}{|c|}{ COEFICIENTES* } & CME \\
\hline & & $a$ & $b$ & $R^{2}$ & \\
\hline $1 \mathrm{~A}$ & $\mathrm{AF}=\mathrm{a}+\mathrm{bLh}$ & $25,72( \pm 1,208)$ & $7,134( \pm 0,141)$ & 0,928 & 368,42 \\
\hline $2 \mathrm{~A}$ & $\mathrm{AF}=\mathrm{a}+\mathrm{bAh}$ & $30,41( \pm 1,209)$ & $13,82( \pm 0,254)$ & 0,937 & 306,32 \\
\hline $3 \mathrm{~A}$ & $\mathrm{AF}=\mathrm{a}+\mathrm{b}(\mathrm{LhXAh})$ & $1,33( \pm 0,439)$ & $0,804( \pm 0,010)$ & 0,97 & 235,56 \\
\hline $1 \mathrm{E}$ & $\mathrm{AF}=\mathrm{a}+\mathrm{bLh}$ & $57,92( \pm 2,409)$ & $6,08( \pm 0,114)$ & 0,935 & 1550,13 \\
\hline $2 \mathrm{E}$ & $\mathrm{AF}=\mathrm{a}+\mathrm{bAh}$ & $31,84( \pm 1,589)$ & $26,35( \pm 0,388)$ & 0,959 & 1410,56 \\
\hline $3 \mathrm{E}$ & $\mathrm{AF}=\mathrm{a}+\mathrm{b}(\mathrm{Lh} x A h)$ & $8,312( \pm 0,801)$ & $0,72( \pm 0,009)$ & 0,971 & 1161,08 \\
\hline $1 \mathrm{Q}$ & $\mathrm{AF}=\mathrm{a}+\mathrm{bLh}$ & $52,06( \pm 1,735)$ & $7,85( \pm 0,129)$ & 0,949 & 696,49 \\
\hline $2 \mathrm{Q}$ & $\mathrm{AF}=\mathrm{a}+\mathrm{bAh}$ & $54,55( \pm 2,446)$ & $28,47( \pm 0,647)$ & 0,907 & 887,09 \\
\hline $3 \mathrm{Q}$ & $\mathrm{AF}=\mathrm{a}+\mathrm{b}(\mathrm{Lh} \times A h)$ & $3,226( \pm 0,908)$ & $1,054( \pm 0,012)$ & 0,955 & 643,31 \\
\hline
\end{tabular}

*a, es la constante, b el coeficiente de regresión lineal, $\mathrm{R}^{2}$ el coeficiente de determinación y CME el cuadrado medio del error expresado en $\mathrm{cm}^{2}$. Los números entre paréntesis corresponden al error estándar de cada coeficiente. 300 hojas por cada especie, se usaron para los cálculos del área foliar.

\section{RESULTADOS Y DISCUSIÓN}

Los datos de los parámetros foliares variaron de la siguiente forma: A. acuminata presentó un largo promedio de $8,38 \mathrm{~cm}$, un ancho promedio de $4,67 \mathrm{~cm}$, con variación entre 4,8 y $12,4 \mathrm{~cm}$ y 2,5 a $7,2 \mathrm{~cm}$. Para $E$. pendula, el promedio de largo de la lámina foliar fue de $20,69 \mathrm{~cm}$, con variaciones de 12,5 y $28,3 \mathrm{~cm}$, mientras que el ancho promedio fue de $3,78 \mathrm{~cm}$ y variaciones entre 1,8 y $5,7 \mathrm{~cm}$. Q humboldtii presentó promedios para el largo de la lámina foliar, sin pecíolo, de 13,03, con variaciones entre 7,3 y $19,8 \mathrm{~cm}$, con ancho promedio de $3,68 \mathrm{~cm}$ y variaciones entre 1,9 y $5,5 \mathrm{~cm}$. La relación alométrica largo por ancho fue de 5,5:1, para E. pendula; 3,5:1, para $Q$. humboldtii y $1,8: 1$, para A. acuminata. Los anteriores datos reflejan las formas particulares de cada especie: A. acuminata, con forma más circular de hoja; E. pendula con forma alargada y Q. humboldtii, de forma intermedia.

De acuerdo a la tabla 1, los nueve modelos probados presentaron coeficientes de determinación superiores a 0,9 y solamente $1 A$ y $2 Q$ fueron inferiores a 0,93 , todos con alto nivel de significancia estadística para la regresión ( $\mathrm{p}<0,001)$, tal y como lo expresa Bozhinova (2006). Lo anterior demuestra que para las tres especies es posible utilizar solo una medición, ya sea el ancho o el largo de su lámina foliar, para estimar, con alto nivel de confiabilidad, el área foliar total; sin embargo, cuando se multiplica el ancho por el largo, los coeficientes son superiores y permiten tener mayor grado de exactitud y de precisión en las mediciones. Esta respuesta coincide con lo encontrado por Cittadini $\mathcal{E}$ Peri (2006), en cerezas, Singh (2007) en guayaba y Nautival et al. (1990), en manzanas, plantas que tiene hojas con formas similares a las especies evaluadas en la presente investigación. Una limitante a la rapidez del método sería el tiempo que se necesita para realizar la medición, pues no solo debe ser medido un atributo, sino los dos y, en ocasiones, como en el caso de $Q$. humboltii, el ancho foliar máximo es difícil de encontrar rápidamente y se necesita promediar varias mediciones.

Para las tres especies, se seleccionaron los modelos tipo $A F=a+b(L h x A h)$. En el caso de $Q$. humboldtii, los modelos $1 Q$ y $3 Q$ (Tabla 1 ), presentaron $\mathrm{R}^{2}$ similar, pero el modelo que contempla solamente el largo de la lámina foliar tiene un error estándar mayor. 
De los tres modelos, $3 E$ fue el que presentó un menor valor en el error estándar de $\mathrm{b}_{1}$, un aspecto muy importante, pues es posible que la amplitud de los valores $\mathrm{X}$, en este caso, el producto de largo por ancho de la hoja, sea mayor y, por tanto, el estimado tenga alta dispersión y mayor grado de confiabilidad, de acuerdo a lo indicado por Samuels E Witmer (2003). O que la desviación estándar de la media sea baja, lo cual, redunda en un alto grado de precisión a la hora de capturar los datos.

De cualquier forma, los componentes de las ecuaciones derivadas del modelo que integra el producto del largo por el ancho de la lámina foliar (LhxAh) son similares a los establecidos por otras investigaciones. La pendiente (b) para A. acuminata fue de 0,80 , para $E$. pendula 0,72 y para $Q$. humboldtii 1,05, mientras que en Actinidia deliciosa, Mendoza de Gyves et al. (2007) encontraron un coeficiente de 0,80, y de 0,97 a 1,1 en Amaranthus caudatus (Ajayi, 1990). Valores 0,71, en manzanas (Nautival et al. 1990) y 0,69 en hojas de Capsicum annuum (De Swart et al. 2004), datos que confirman la utilidad del método en hojas con formas similares a las especies trabajadas en este estudio.

En síntesis, se deduce por los datos estadísticos, que los siguientes modelos matemáticos pueden ser usados para calcular el área foliar de forma no destructiva: para $A$. acuminata $A F=1,333+0,84($ LhxAh); para $E$. pendula $\mathrm{AF}=8,312+072(\mathrm{LhxAh})$ y para $Q$. humboldtii $\mathrm{AF}=-3,226+1,054$ (LhxAh). Los coeficientes de determinación $\left(\mathrm{R}^{2}\right)$ para cada caso fueron 0,$97 ; 0,97$ y 0,96 . Valores similares fueron publicados por otros investigadores, para estimar el área individual de la hoja, con el uso del producto largo por ancho de la misma. Mendoza de Gyves et al. (2007 reportaron un valor $\mathrm{R}^{2}$ de 0, 98 para hojas de A. deliciosa, Singh (2007) 0,96 en hojas de Psidium guayaba, mientras que Bange et al. (2002) encontraron un $R^{2}$ de 0,97 en hojas de Helianthus annus.

Al analizar las gráficas obtenidas de los tres modelos escogidos (Figura 1), se observa que $E$. pendula presenta mayor amplitud en los datos correspondientes a la variable independiente $(\mathrm{X})$, lo que indica mayor grado de confianza al tomar mediciones ya sea en hojas pequeñas o con tamaño muy superior al promedio. Debido a esto, el error estándar de la pendiente es menor para especie y el coeficiente de determinación fue el más alto de todos los modelos.

La comparación entre los datos medidos y los datos estimados del área de la hoja de las tres especies, mediante el modelo $A F=a+b(L h x A h)$, produjo un alto coeficiente de correlación $\left(\mathrm{r}^{2}\right)$ entre las dos técnicas para las tres especies, el cual, fue de 0,99 , en todos los casos, mientras que se presentaron altos niveles de significancia para la prueba de comparación de medias $(p<0,001)$. La validación del modelo es un paso importante para asegurar las implicaciones de la ecuación producida para predecir valores del área de las hojas, a través de mediciones rápidas y de bajo costo. Este aspecto es de gran importancia, especialmente, en plantas perennes, donde el muestreo destructivo limita, en muchas ocasiones, el seguimiento en el tiempo de procesos fisiológicos como la fotosíntesis foliar, la respiración, el crecimiento de órganos, el uso eficiente del agua y el uso eficiente de la radiación solar. La variación explicada para los tres modelos, entre lo observado y lo predicho, fundamenta el uso de las fórmulas con seguridad y precisión (Tabla 2).

En las figuras 2, 3 y 4, se observa la relación entre de los valores estimados y los valores medidos directamente del área individual de las hojas de $A$. acuminata, E. pendula y Q. humboldtii. Algunas investigaciones validan lo aquí expresado. Demirsoy et al. (2005) encontraron $\mathrm{r}^{2}$ de 0,99, para área de hojas en fresa y Mendoza de Gyves et al. (2007) hallaron un $\mathrm{r}^{2}$ de 0,993 en A. deliciosa, mientras que Rouphael et al. (2006) obtuvieron un $\mathrm{r}^{2}$ de 0,98 en Cucurbita pepo var suchini. El alto coeficiente de correlación en los tres casos y la alta significancia estadística de la prueba, permiten establecer con certeza y con exactitud, que los tres modelos seleccionados pueden ser utilizados en trabajos de campo, con alta precisión matemática. 


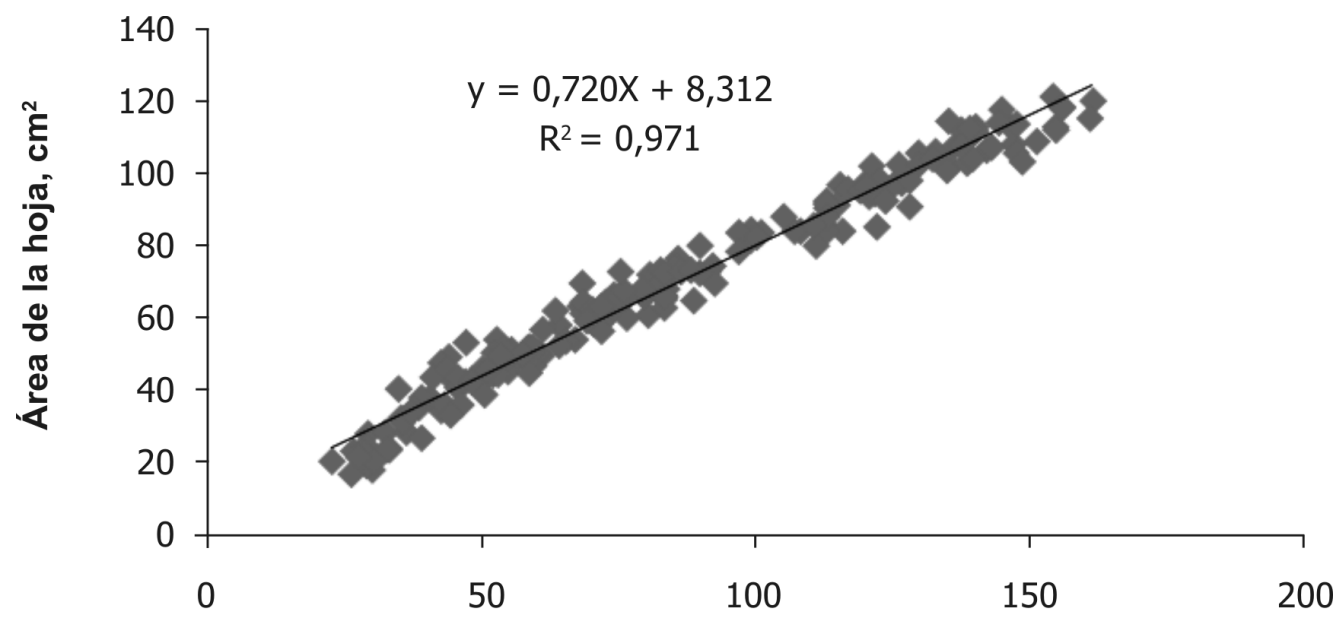

Producto de ancho por largo de la hoja, $\mathrm{cm}^{2} \quad \mathrm{~A}$
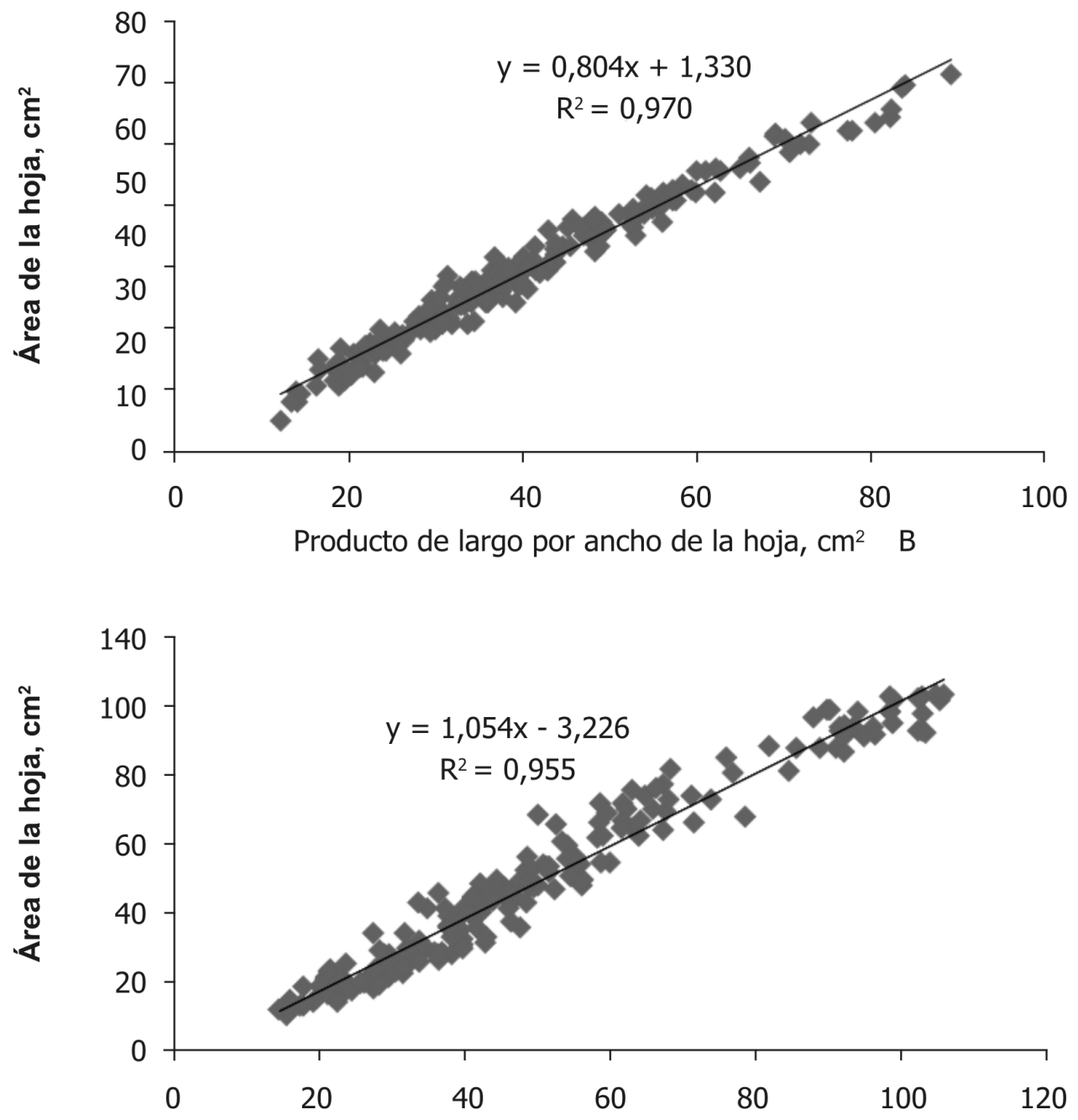

Producto de ancho por largo de la hoja, $\mathrm{cm}^{2} \quad \mathrm{C}$

Figura 1. Relación entre el producto largo por ancho y el área foliar individual, en hojas de Alnus acuminata (A), Escallonia pendula (B) y Quercus humboldtii (C). 
Tabla 2. Análisis de regresión para los valores estimados y valores medidos del área de hojas individuales de $A$. acuminata, E. pendula y $Q$. humboldtii, a partir del modelo $A F=a+b(A h x L h)$.

\begin{tabular}{|c|c|c|c|c|c|c|}
\hline \multicolumn{7}{|l|}{ A. acuminata } \\
\hline Fuente & G.L & C. Medio & F.C & Significancia & E.E. & $R^{2}$ \\
\hline Regresión & 1 & 28434,68 & 7741 & $* * *$ & 1,9165 & 0,9875 \\
\hline Residual & 98 & 3,6731 & & & & \\
\hline Total & 99 & & & & & \\
\hline \multicolumn{7}{|l|}{ E. pendula } \\
\hline Fuente & G.L & C. Medio & F.C & Significancia & E.E & $\mathrm{R}^{2}$ \\
\hline Regresión & 1 & 71947,39 & 9870,48 & $* * *$ & 2,6998 & 0,99 \\
\hline Residual & 98 & 7,289 & & & & \\
\hline Total & 99 & & & & & \\
\hline \multicolumn{7}{|l|}{ Q. humboldtii } \\
\hline Fuente & G.L & C. Medio & F.C. & Significancia & E.E & $\mathrm{R}^{2}$ \\
\hline Regresión & 1 & 54647,88 & 8559,4 & $* * \star$ & 2,4836 & 0,989 \\
\hline Residual & 98 & 6,1683 & & & & \\
\hline Total & 99 & & & & & \\
\hline
\end{tabular}

*error estándar de la media.

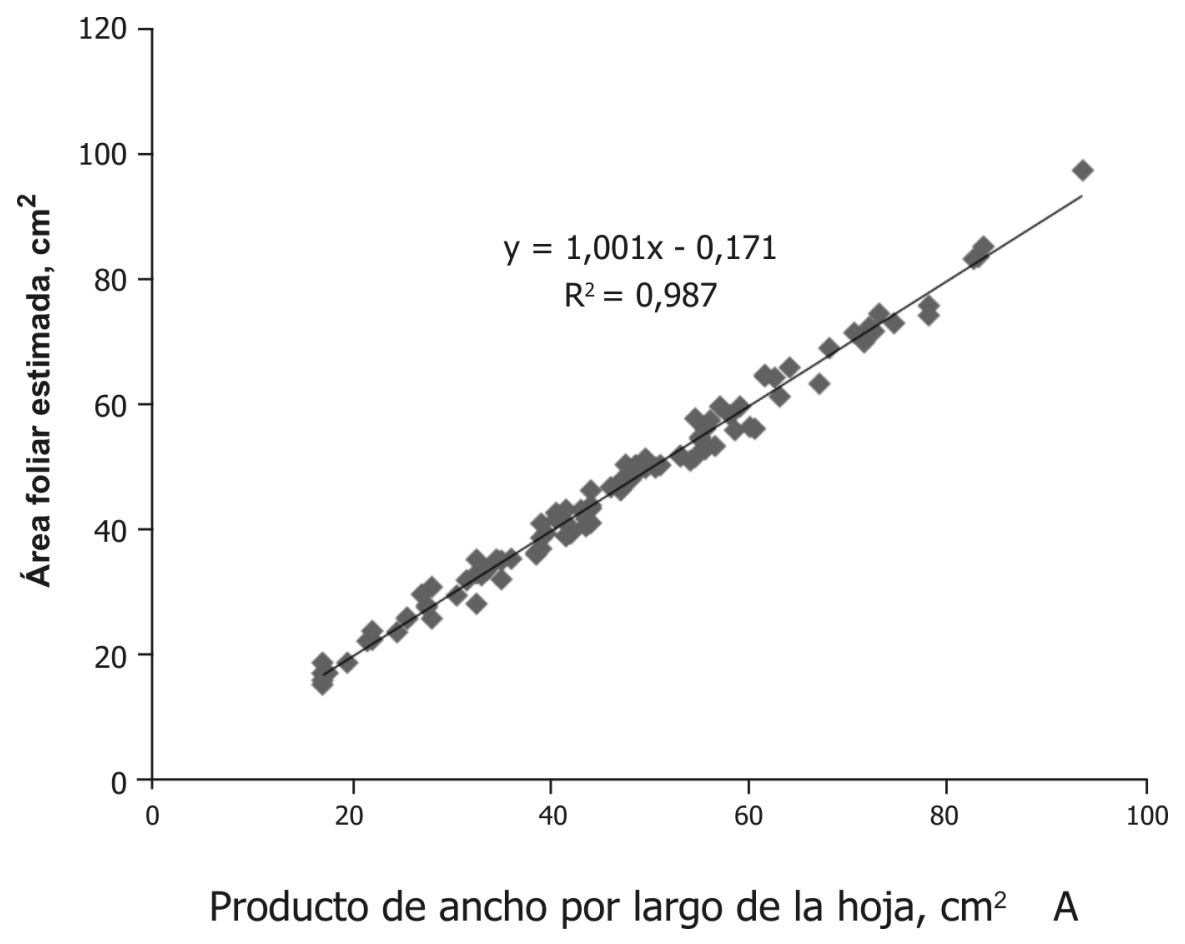

Figura 2. Relación entre el área foliar estimada y el área foliar, medida en hojas de Alnus acuminata. 


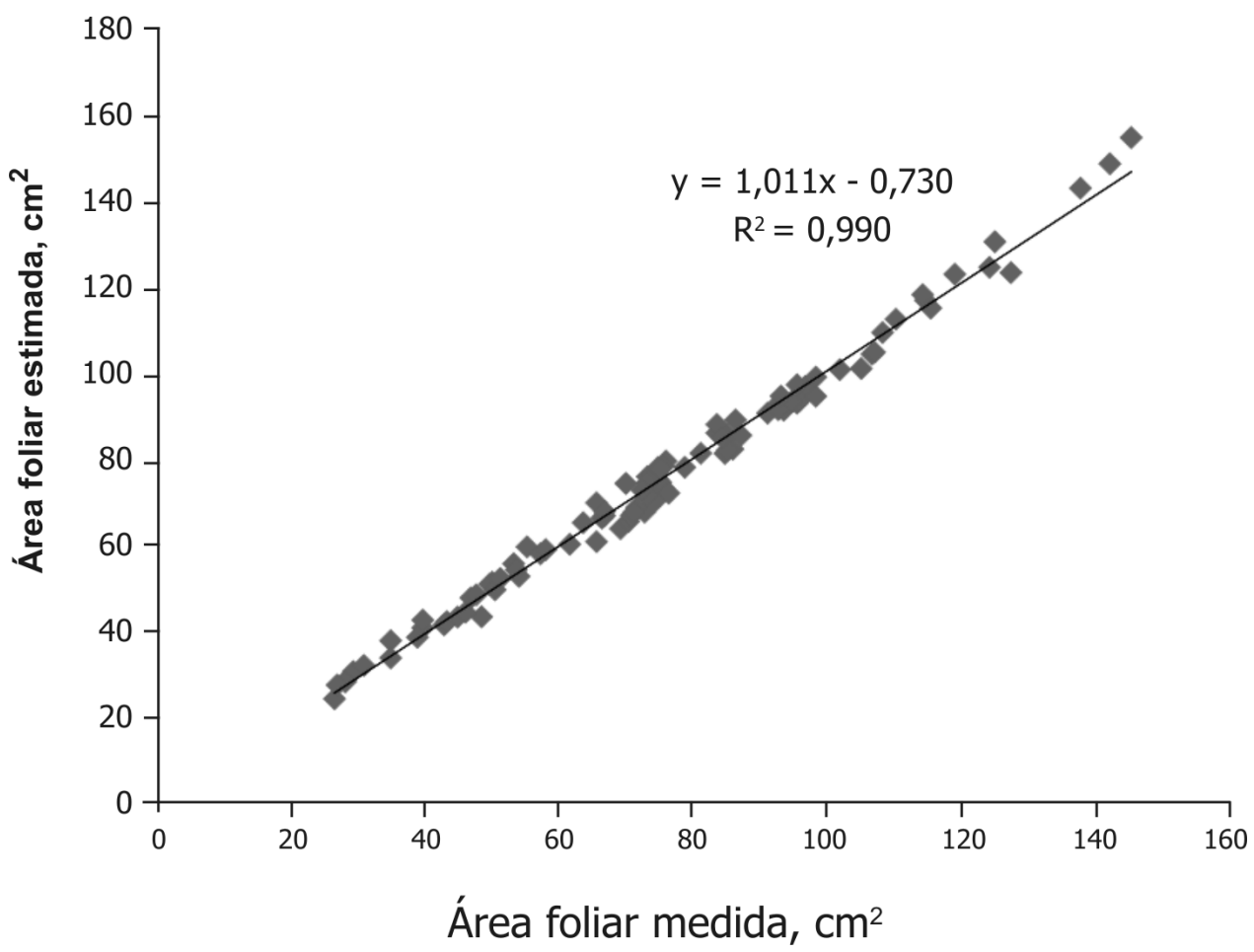

Figura 3. Relación entre el área foliar estimada y el área foliar, medida en hojas de Escallonia pendula.

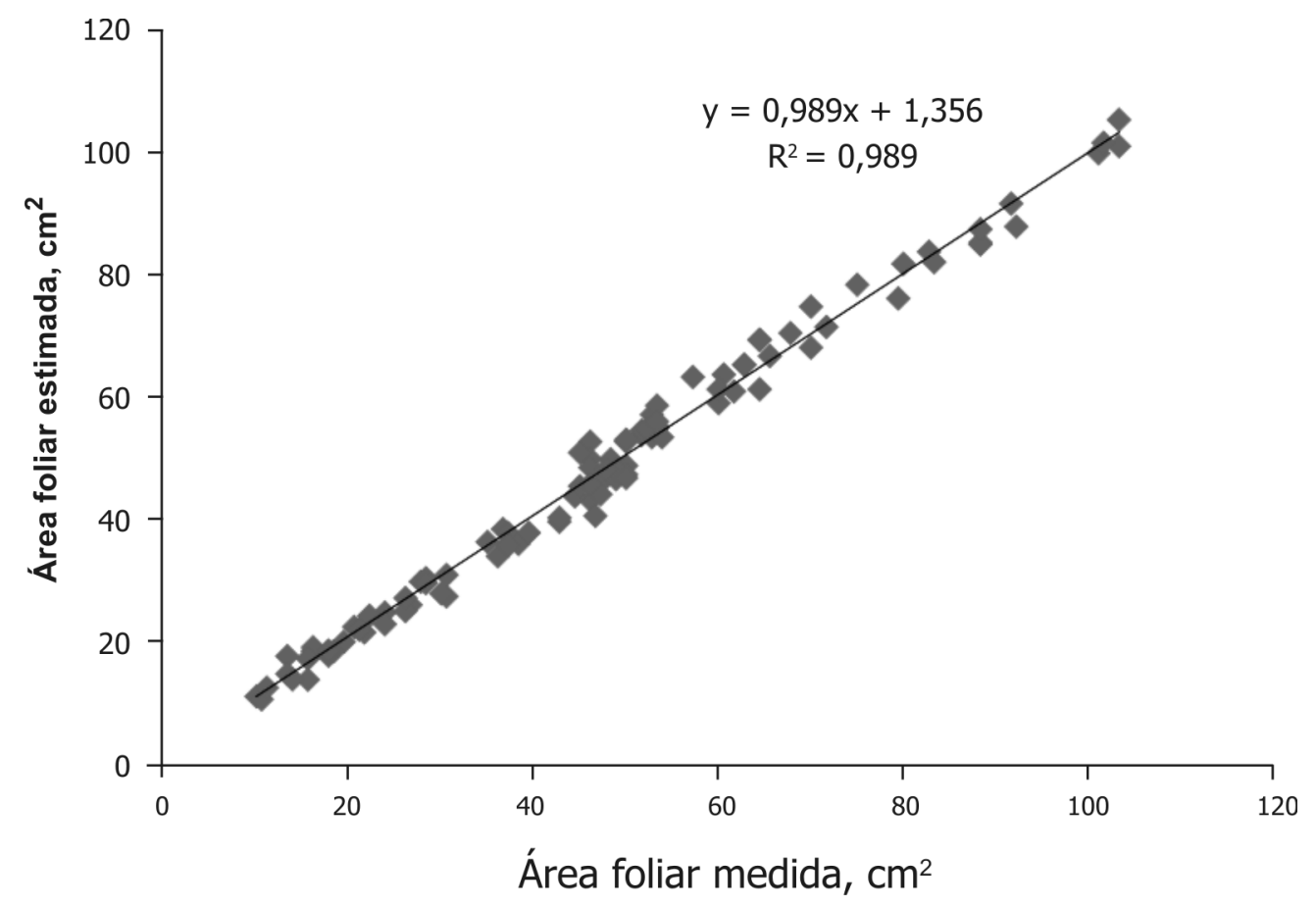

Figura 4. Relación entre el área foliar estimada y el área foliar, medida en hojas de Quercus humboldtii. 


\section{CONCLUSIONES}

Bajo las condiciones del presente ensayo, el modelo que utiliza las medidas de longitud y ancho de las hojas, producto de la multiplicación de los dos atributos foliares, puede proveer estimaciones de alta precisión para la determinación rápida y económica del are foliar en plantas de A. acuminata, E. pendula y $Q$ humboldtii, Las ecuaciones propuestas en la presente investigación pueden ser empleados con seguridad en estudios fisiológicos, biológicos, ambientales, agronómicos, ecológicos y forestales, sin necesidad de recurrir al muestreo destructivo.

Se demuestra que esta técnica es una herramienta muy útil para realizar estudios de vegetación en zonas donde no se pueda tener acceso a tecnología de punta, para realizar mediciones electrónicas.

Es posible también usar uno de los dos atributos de la hoja, ya sea el largo o el ancho de las mismas, especialmente, en $A$. acuminata y $E$. pendula, para estimar el área de la hoja. Esto reduce el tiempo de medición y facilita el manejo de la información. Queda en manos de los investigadores el empleo de los modelos, dependiendo de los objetivos y las condiciones del trabajo.

\section{BIBLIOGRAFÍA}

AJAYI, N.O. 1990. Rapid determination of leaf area in ovate vegetable leaves by linear measurements. J. Hort. Sc. 65(1):1-15.

AKRAM-GHADERI, M.; SOLTANI, F.A.. 2007. Leaf area relationships to plant vegetative characteristics in cotton (Gossypium hirsutum L.) grown in a temperate subhumid environment. Internal. J. Plant. Prod. 1(1): 63-71.

ARCILA, P.J. 1991. Métodos rápidos para la determinación de área foliar de cafeto en campo. En: XXI Congreso anual y Primer Simposio Nacional sobre Fisiología de la Nutrición Mineral. Manizales, 20, 21 y 22 de febrero, 77p.

BANGE, M.P.; HAMMER, G.L.; MILROY, S.P.; RICKERT, K.G. 2000. Improving estimates of individual leaf area of Sunflower. Agron. J. 92:761-765.
BEERLING, D.J.; FRAY, J.C. 1990. A comparison of the accurate variability and speed of five different methods for estimating leaf area. Ann. Bot. 65:483-488.

BOZHINOVA, R.P. 2006. Coefficients for determination of the leaf area in three burley. J. Central Eur. Agric. 7(1):7-12.

BROADHEAD, J.S.; MUXWORTHY, A.R.; ONG, C.K.; BLACK, C.R. 2003. Comparison of methods for determining leaf area in tree rows. Agric. Forest. Meteorol. 115:151-161.

CITTADINI, E.D.; PERI, P.L. 2006. Estimation of leaf area in sweet cherry using a non-destructive method. RIA. 35(1):143-150.

CRAIG MACFARLANE, C.; HOFFMAN, M.; EAMUS, D.; KERP, N.; HIGGINSON, S.; MCMURTRIE, R.; ADAMS, M. 2007. Estimation of leaf area index in eucalypt forest using digital photography. Agric. Forest. Meteorol. 143:176-188.

DEMIRSOY, H.; DEMIRSOY. L.; ÖZTÜRK, A. 2005. Improved model for the non-destructive estimation of strawberry leaf area. Fruits. 60:69-73.

DE SWART, E.A.M.; GROENWOLD, R.; KANNE, H.J.; STAM, P.; MARCELLIS, L.F.M.; VOORRIPS, R.E. 2004. Non-destructive estimation of leaf area for different plant ages and accessions of Capsicum annum L. J. Hortic. Sci. Biotec. 79:764-770.

ERIKSSON, H.; EKLUNDH, L.; HALL, K.; LINDROTH, A. 2005. Estimating LAI in deciduous forest stands. Agric. Forest Meteorol. 129:27-37.

GUTEZEIT, B. 2000. Non-destructive measurement of fresh plant mass by the gamma-scanning technique applied to broccoli. J. Agric. Engng. Res. 75:251-255.

GUTIÉRREZ, T.A.; LAVÍN, A.A. 2000. Mediciones lineales en la hoja para la estimación no destructiva del área foliar en vides cv. Chardonnay. Agric. Téc. 60(1):9-17.

JONCKHEERE, I.; FLECK, S.; NACKAERTS, K.; MUYS, B., COPPIN, P.; BARET, F. 2004. Review of methods 
for in situ leaf area index determination Part I. Theories, sensors and hemispherical photography. Agric. Forest Meteorol. 121:19-35.

KUCHARIK, CH.J.; NORMAN, J.M.; GOWER, S.T. 1998. Measurements of branch area and adjusting leaf area index to indirect measurements. Agric. Forest Meteorol. 91:69-88.

LEVY, P.L.; JARVIS, P.G. 1999. Direct and indirect measurements of $\mathrm{LAI}$ in millet and fallow vegetation in HAPEX-Sahel. Agric. Forest. Meteorol. 97:199212.

MENDOZA DE GYVES, M.; ROUPHAEL, Y.; CRISTOFOTI, V.; MIRA, F.R. 2007. A non-destructive simple and accurate model for estimating the individual leaf area of kiwi (Actinidia deliciosa). Fruits. 62:171175.

NAUTIVAL, B.M.; SINGH, P.K.; SUKLA, R.N.; PRAKASH, S.; KUMAR, A. 1990. Correcting leaf area measurement by conventional methods: a new approach for apple (Malus domestica Borkh). J. Hort. Sc. 65(1):15-18.

PANTA, G.R.; NeSMITH, D.S. 1994. A model for estimating area of muskmelon leaves. HortScience. 30:624-625.

PEKSEN, E. 2007. Non-destructive leaf area estimation model for faba bean (Vicia faba L.). Scientia Hort. 113:322-328.
PEPER, P.J.; McPHERSON, E.G. 2003. Evaluation of four methods for estimating leaf area of isolated trees. Urban For. Urban Green. 2:19-29.

ROBERTS, S.T.; EVANS, TH.L.; McCOMBS, J.W.; HARRINGTON, R.L.; GLASS, P.A. 2005. Estimating individual tree leaf area in loblolly pine plantations using LiDAR derived measurements of height and crown dimensions. Forest. Ec. Man. 213:54-70.

ROSS, J. 1981. The radiation regime and architecture of plant stands. Dr. W. Junk Publischer, The Hague, Ned. 392p.

ROUPHAEL, Y.; RIVERA, C.M.; CARDARELLI, M.; S. FANASCA, S.; COLLA, D. 2006. Leaf area estimation from linear measurements in zucchini plants of different ages. J. Hortic. Sci. Biotec. 81(2):238-241.

SAMUELS, M.J.; WITMER, J.A. 2003. Statistics for the life sciences. $3^{\text {rd }}$ ed. Pearson Education Inc. N. J. p.525-594.

SINGH, A. 2007. Approximation of leaf area by using leaf dimensions in guava. I International Guava Symposium. Acta Hort. 735:321-324.

SONNENTAG, O., TALBOT, J.; CHEN, J.M.; ROULET, N.T. 2008. Using direct and indirect measurements of leaf area index to characterize the shrub canopy in an ombrotrophic peatland. Agric. Forest. Meteorol. 144:200-212.

Recibido: Septiembre 12 de 2008

Aceptado: Marzo 27 de 2009 